\title{
STUDY OF AN EFFECT OF INELASTIC DEFORMATION ON THE HEAT CAPACITY OF STEEL
}

\author{
Maxim Zhelnin, Anastasiya Iziumova, Aleksei Vshivkov, Oleg Plekhov ${ }^{+*}$ \\ Institute of Continuous Media Mechanics of the Ural Branch of Russian Academy of Science, 1 Academika \\ Koroleva str., Perm 614013, Russia \\ ${ }^{+*}$ Presenting and Corresponding Author: poa@icmm.ru
}

\begin{abstract}
Based on the technique for determination of thermal constants in metals we have determined the heat conductivity and heat capacity of metal samples. The technique was adapted for application during standard mechanical test to determine the evolution of thermo mechanical properties of the samples under quasistatic loading. It gives us an opportunity to define the effect of inelastic deformation on the thermal constants of metals using infrared data treatment. The progress in the precision of proposed technique opens very promising perspectives for experimental measurements of thermo mechanical properties (for instance, configuration entropy) of deformed metals.
\end{abstract}

KEYWORDS: Inelastic deformation, heat capacity, infrared thermography.

\section{INTRODUCTION}

Heat conductivity is one of the important properties allowing assessment not only possibility to use of material in a given conditions but to obtain information about its mechanical properties. To measure the real value of heat conductivity and heat capacity is important both for correct measurement of defect induced dissipated energy value and for study of the structure evolution in metals under plastic deformation.

The dissipated energy study allows us to validate the energy criterion of the fracture. These criteria $[1,2]$ are based on assessment of proportion between dissipated energy and deformation energy. In this case, real values of thermal constants of materials are necessary to solve the heat conductivity problem and to evaluate power of heat dissipation during deformation of materials under quasistatic and cyclic loading conditions [3,4].

The study the effect of inelastic deformation on the value of thermal constants allows us to quantify the current stage of structure evolution and give the promising way to experimentally validate the thermo dynamical potential of the system. The progress in the precision such techniques opens the perspectives for experimental measurements of thermo mechanical parameters of the system (for instance, configuration entropy) and gives important information for development of the constitutive equations for inelastic metal deformation.

Nowadays there are a lot of techniques to measure thermal constants of materials. In general, these techniques could be divided into two types: steady-state and transient methods. The first one is used to determine thermal conductivity directly and required relatively large specimen and long measuring times. The second one uses small specimen, and the measuring time is a few minutes but this type of techniques allows to measure thermal diffusivity only [5]. The main feature of presented technique is possibility to determine the heat conductivity and heat diffusivity simultaneously during the same experiment and study the effect of inelastic deformation on the measurement parameters.

\section{THEORETICAL BACKGROUND}

The temperature field evolution can be described by a three-dimension heat conduction equation:

$$
\frac{\partial T}{\partial t}=\alpha \Delta T,-\infty<x, y<+\infty, 0<z<l
$$

with corresponding initial and boundary conditions.

The Carslaw and Jaeger solution at the point $(0,0, l)$ can be written in the following form[6]:

$$
T(t)=\frac{q}{4 \lambda l}\left[\ln \left(\frac{4 \alpha}{\omega^{2}} t+1\right)+2 \sum_{n=1}^{+\infty}(-1)^{n} \exp \left(\frac{\pi^{2} n^{2} \omega^{2}}{4 l^{2}}\right) \cdot\left(\Gamma\left[0, \frac{\pi^{2} n^{2} \omega^{2}}{4 l^{2}}\right]-\Gamma\left[0, \frac{\pi^{2} n^{2}\left(4 \alpha t+\omega^{2}\right)}{4 l^{2}}\right]\right)\right]
$$


where $\Gamma(x)=\int_{0}^{+\infty} t^{x-1} e^{-t} d t$ is the gamma function, $\alpha$ is the thermal diffusivity, $\lambda$ is the thermal conductivity, $q$ is thermal flux capacity, $\omega$ is a distance at which the thermal flux capacity reduced by a factor of $1 / e^{2}$.

To define the thermal diffusivity $\alpha$ and the thermal conductivity $\lambda$ we solve the optimization problem ( $\alpha$ and $\lambda$ are optimization parameters) and find the best correspondence between experimentally obtained temperature evolution of heated point and theoretical solution.

\section{EXPERIMENTAL CONDITIONS, DATA PROCESSING AND RESULTS}

Experimental determination of the thermal diffusivity and the thermal conductivity was carried out on stainless steel samples (AISI 304). Before starting the experiment, the polished surface was covered by a thin layer of matte black paint. The temperature evolution of front specimen surface was recorded by infrared (IR) camera FLIR SC 5000 at the front specimen surface. The rear surface of the specimen was heated by a contact heat source. Noise filtration was made by Gaussian filter.

The specimen was placed in the grips of servo-hydraulic machine Bi-00-100. In order to determine the coefficients $q, \omega$ of the heat source, we have performed measurements on the unstrained specimen. Further, the specimen was stretched to given strain levels: $12 \%, 24 \%, 36 \%, 41 \%$. A rate of test machine pistons was $0.2 \mathrm{~mm} / \mathrm{s}$. The thermal diffusivity $\alpha$, the thermal conductivity $\lambda$ and the thickness $l$ of the specimen were determined at the each strain level. Based on the assumption of a small variation of a density of the material during deformation, the heat capacity at the each strain level was determined by using the relation $\lambda /(\rho \cdot a)$, where $a, \lambda$ are the thermal diffusivity and the thermal conductivity at the given strain level, and the density $\rho$ is unchanged.

Figure 1 presents dependence the heat capacity of the stainless steel AISI 304 on the deformation. As can be seen from the figure, an increase in the deformation led to a decrease in the heat capacity of the steel. Thus based on the data presented in fig. 1 we can conclude that there is a linear relation between the deformation and the heat capacity.

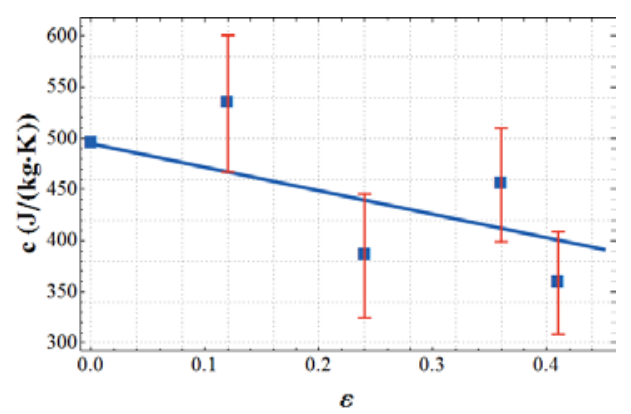

Fig. 1 A dependence of the heat capacity of the stainless steel AISI 304 on the deformation.

\section{CONCLUSIONS}

As a result, the infrared measurements have been used to investigate thermal diffusivity, the thermal conductivity and the heat capacity of metals based on the proposed technique. Developed technique allows one to determine heat diffusivity and heat conductivity simultaneously from the same experiment. Obtained values are in a satisfactory agreement with data of thermal diffusivity and thermal conductivity obtained in another literature source [7].

The study of the influence of the deformation on the heat capacity of stainless steel presented in this paper allows one to conclude, that an increase in the deformation led to a decrease in the heat capacity of the steel.

\section{REFERENCES}

[1] A.A. Griffith, Phil. Trans. Roy. Soc., ser. A. 221 (1920) 163-198.

[2] V. V. Fedorov, Damage and fracture kinetics of solids, Tashkent, «FAN» Uz SSR. (1979). 1- 168.

[3] A.Yu. Iziumova, A. N. Vshivkov, A. E. Prokhorov, O. A. Plekhov and B. Venkatraman, PNRPU Mechanics Bulletin 1, (2016) 68-84.

[4] A. Fedorova, M. Bannikov, A. Terekhina and O. Plekhov, QIRT journal. 11 (2014) 2-9.

[5] Tseng-Wen Lian et all, Advanced Powder Technology 1. (2016) 1-4.

[6] H. S. Carslaw and J. C. Jaeger, Conduction of heat in solids, Clarendon Press, Oxford. (1959) 1-510.

[8] U. B. Egorova, E. N. Egorov and R. A. Davidenko, Avtomobilnaya promishlennost. 12 (2011) 30-33. 